\title{
Total Tissue
}

National Cancer Institute

\section{Source}

National Cancer Institute. Total Tissue. NCI Thesaurus. Code C90476.

The total collection of tissues within a sample. 